\title{
Research on Duffing Oscillator Algorithm of Secondary Traffic Accident Xinglei Zhang ${ }^{\text {a }}$, Wenhui Zhang ${ }^{\text {b }}$ \\ Northeast forestry university traffic college, Harbin 150040, China \\ azhang_xinglei321@126.com, ${ }^{b}$ rayear@163.com
}

Keywords: Secondary traffic accidents; Chaos characteristic; Duffing equation; Simulation.

\begin{abstract}
In order to discover evolution characteristics of secondary traffic accidents and prevent from secondary traffic accidents, chaos characteristics of secondary traffic accidents are analyzed based on describing Duffing oscillator algorithm. Afterwards, chaos model is established. By means of simulation, parameters values in chaos model are changed to obtain system constructions when periodic oscillation and chaos state occur. The phase plane and root locus diagrams are drawn accordingly. Through case study, proof of Duffing oscillator model is available for secondary analysis of the evolution of the traffic accident, the simulation results can be provided a theoretical basis for secondary traffic accident prevention measures.
\end{abstract}

\section{Introduction}

After traffic accidents occur, it is essential to wait for policemen closing in accident scenes. Objective existence of accident scenes induce to driveway closing. Accordingly, the drivers of passing vehicles need to change driving behavior. Traffic flow parameters will change, such as space headway, average velocity. Safety of section in traffic accident scenes is worst. If drivers judge or operate mistakenly, the passing vehicles are likely to crash into accident scenes. Secondary traffic accidents possibly happen. As continued accidents of first ones, secondary traffic accidents result to more serious casualty and property loss[1,2].

It is shown that causation analysis for secondary traffic accidents is few by Consulting literature. It is owing to statistical data of secondary traffic accident is lack. Consequently, quantitative models depended on statistical data are improper. Chaos theory can be used to study random and unpredictable phenomenon of nonlinear system. Since Lorenz in America found chaos phenomenon in 1963, chaos theory has developed to be applied to predict[3-5] and control nonlinear system[6,7]. Based on chaos theory, this paper analyzes chaos characteristics of secondary traffic accident. Afterward, Duffing vibrator model is established. The model is simulated to analyze the causation of secondary traffic accident in defect of statistical data.

\section{Duffing Vibrator Model}

P.Halmes presented Duffing Vibrator model based on random vibration in 1979[8]. As shown in Figure1, the elastic thin steel was fixed on rigid frame, under which two magnets were set. When the frame made simple harmonic vibration, dynamic equation of steel was Duffing Vibrator model. Owing to the actuation of vibration and magnetic force, the motion of steel was featured chaos state.

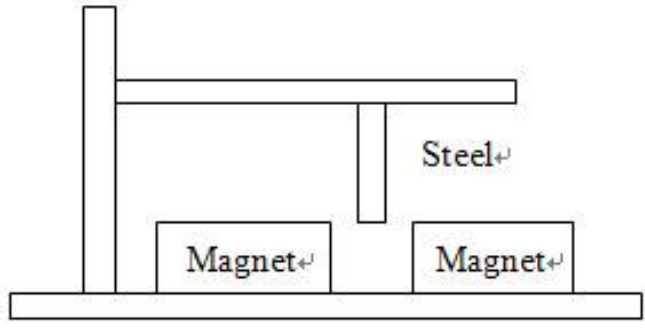

Fig. 1 Simulation of Chaos Vibrator

Dynamic equation of the vibrator could be expressed as follows. 


$$
\mathrm{X}+\frac{\mathrm{D}}{\mathrm{M}} \mathrm{X}-\frac{\mathrm{C}}{\mathrm{M}} \mathrm{X}+\frac{\mathrm{N}}{\mathrm{M}} \mathrm{X}^{3}=\frac{\mathrm{F}}{\mathrm{M}} \cos (\omega \mathrm{T})
$$

$\mathrm{M}$ represented particle weight $(\mathrm{kg})$. D represented damping coefficient. $\mathrm{C}$ and $\mathrm{N}$ represented linear and nonlinear coefficient individually. F represented outside force $(\mathrm{N})$.

Eq. 1 describes synthesis of linear and nonlinear motion. Meanwhile it meant that the steel made nonlinear motion on account of magnetic force. Therefore, the system was nonlinear chaos system. But the system could change from ordered state to disordered state by means of modifying coefficients in Eq. 1.

The parameters of $X=\alpha x$ and $T=\beta t$ were introduced and variable substitution was performed. The simplified dynamic equation was shown as follows.

$$
\ddot{\mathrm{x}}+\mathrm{ax}-\mathrm{x}+\mathrm{bx}^{3}=\gamma \cos (\Omega \mathrm{t})
$$

$a$ and $b$ represented different damping.

\section{Chaos characteristics of secondary traffic accident}

After traffic accident occurs, the passing vehicles, personnel around accident scene, traffic policemen and traffic environment compose of a nonlinear dynamic system. State changing of every component leads safety changing of traffic accident scene. Consequently, occurrence of secondary traffic accident is uncertain. The uncertain characteristic shows random of chaos phenomenon. Furthermore, the sudden characteristic of secondary traffic accident shows dependence of chaos results to initial conditions.

Occurrence of secondary traffic accident has similarity with chaos system, which express as follows.

(1)Both located in nonlinear frame. Chaos motion occurs under setting system frame, that is nonlinear vibrating system. The passing vehicle, personnel, traffic policemen and traffic environment around accident scene also form a nonlinear system.

(2)System construction plays a dominative role during changing. In chaos equation, every coefficient represents degree of nonlinear. And different coefficient affects evolvement to chaos state. For traffic system, section of accident scene is the bottleneck of whole highway. According safety is worst. Harmony of Personnel, vehicle and highway is precondition of safety in accident section. If one of the three factors is maladjusted, construction of traffic system is affected. Therefore the drivers need to change driving status to avoid secondary traffic accident.

(3)Different construction of system produces different evolvement to chaos state. But adjustment of construction can change evolvement process. When coefficients are modified in chaos equation, different chaos process and self-organization phenomena will take place. After traffic accident occurs, traffic diversion can reduce amount of passing vehicles. Limited speed or traffic sign setting reasonably changes construction of traffic system. Hence, safety in traffic accident scene is improved.

According to chaos characteristics of secondary traffic accident, chaos dynamic model is established.

$$
\ddot{Z}+\varepsilon \delta \dot{Z}-Z+Z Z^{3}=\varepsilon \sigma \cos \theta t
$$

In the formula, $Z$ represents state of traffic system, including state of initial accident scene, chaos state and state of secondary accident occurrence. \& and individually represent velocity and acceleration of state changing. $\delta$ represents performance parameter of passing vehicles. $\varepsilon$ represents parameter of traffic environment in accident scene. $\sigma$ represents parameter of drivers. $t$ is variable of time. $\theta$ is parameter of system.

Eq. 3 indicates that traffic environment affects both vehicles performance and driving status, which accords with ergonomics. Differential equation is solved as follows. 


$$
\mathrm{Z}_{\mathrm{j}}=\mathrm{Z}_{\mathrm{j}}(\mathrm{t}) \mid \mathrm{t}=\mathrm{t}_{0}+\int_{\mathrm{t}_{0}}^{\mathrm{t}} \dot{\mathrm{Z}}_{1}(\delta, \varepsilon, \sigma) \mathrm{dt}=\mathrm{Z}_{\mathrm{j}}\left(\mathrm{t}_{0}\right)+\Psi\left(\mathrm{Z}_{j}(\mathrm{t}), \delta, \varepsilon, \sigma\right)
$$

In Eq. $4, Z_{j}\left(t_{0}\right)$ is current safety state that can be predicted. Safety state in accident scene is nonlinear function involving variable of $\psi_{j}\left(Z_{j}\left(t_{0}\right), \delta, \varepsilon, \sigma\right)$. Furthermore, $\psi_{j}\left(Z_{j}\left(t_{0}\right), \delta, \varepsilon, \sigma\right)$ relates to $Z_{j}\left(t_{0}\right), \delta, \varepsilon$ and $\sigma$. Modification of above construction parameters can improve system safety.

\section{Simulation for Duffing Vibrator Model of Secondary Traffic Accident}

Model Simulation. In Eq. 3, when $\varepsilon \delta=0.3, \varepsilon \sigma=0.2, \theta=1$, root locus and phase plane are shown in Fig. 2 and Fig.3.

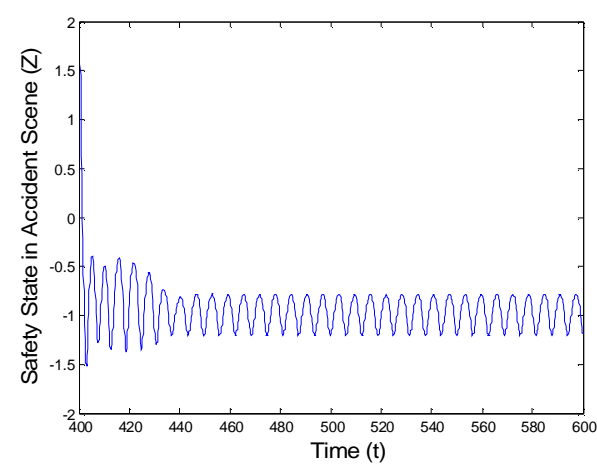

Fig. 2 Root Locus Graph $(\varepsilon \delta=0.3 \varepsilon \sigma=0.2 \theta=1)$

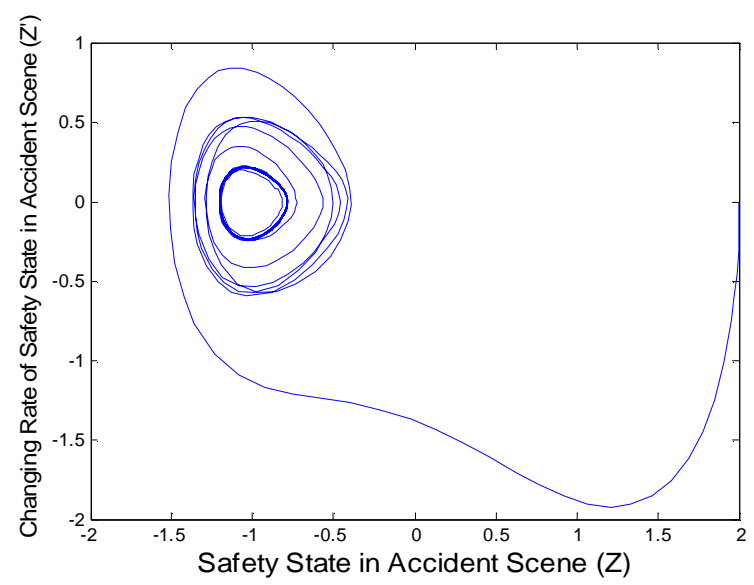

Fig. 3 Phase Plane Graph $(\varepsilon \delta=0.3 \varepsilon \sigma=0.2 \theta=1)$

In Fig. 2 and Fig. 3, it can be seen that safety system of accident scene is in periodical vibration state.

When $\varepsilon \delta=0.3, \varepsilon \sigma=0.5, \theta=1$, root locus and phase plane are shown in Fig. 4 and Fig. 5.

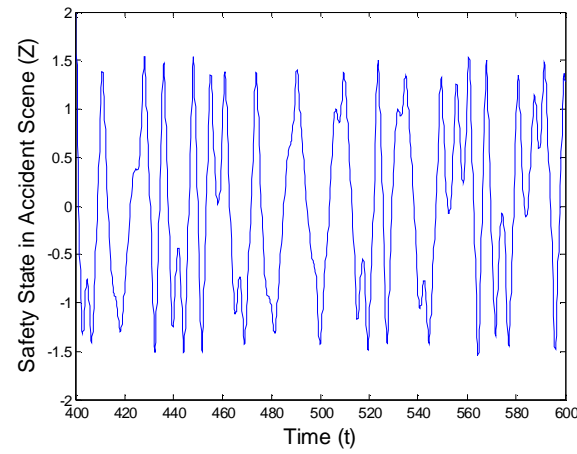

Fig. 4 Root Locus Graph $(\varepsilon \delta=0.3 \varepsilon \sigma=0.5 \theta=1)$

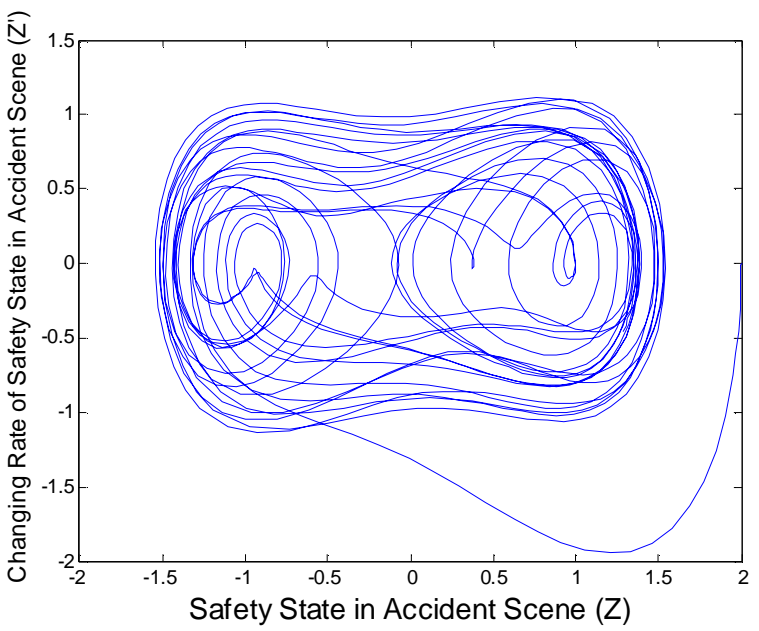

Fig. 5 Phase Plane Graph $(\varepsilon \delta=0.3 \varepsilon \sigma=0.5 \theta=1)$

In Fig. 4 and Fig. 5, it can be seen that safety system in accident scene is in chaos state. Fig. 4 indicates that the amplitude in chaos is larger than that in vibration. Consequently, safety in accident scene is more sensitive, which reflect secondary trafffic accident preventive. If $\delta, \varepsilon$ or $\sigma$ is modified, secondary traffic accident is possible to be avoid.

Example Analysis. According to investigation data of accident scene in reference Eq. 4, the value of coefficients in Eq. 3 can be determined as follows: $\delta=0.1114 ; \varepsilon=0.63282 ; \sigma=0.2504$.

Root locus and phase plane are shown in Fig. 6 and Fig. 7 by means of simulation. 


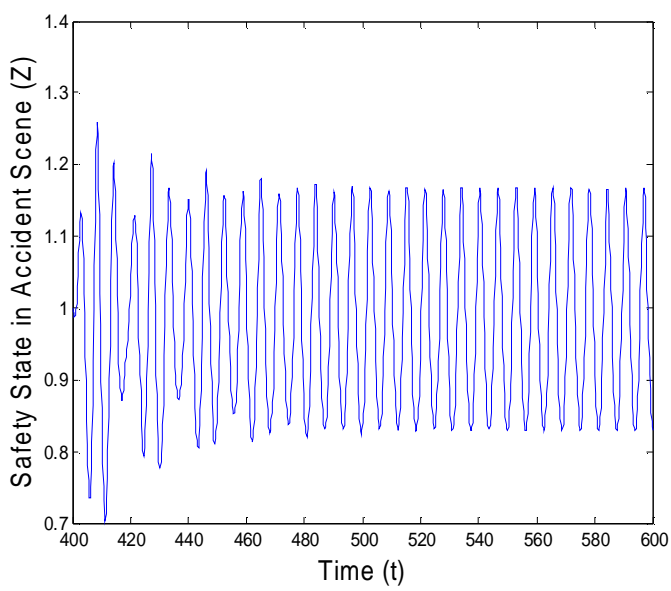

Fig. 6 Root Locus Graph

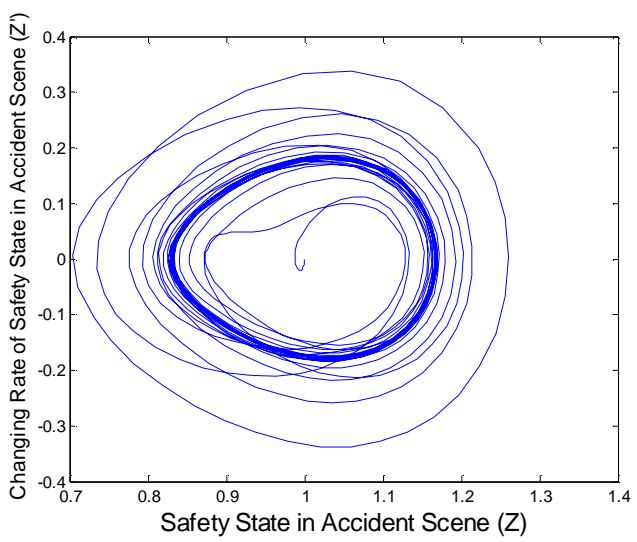

Fig. 7 Phase Plane Graph

From Fig. 6 and Fig. 7 it can be seen that safety system of accident scene is in chaos state and safety state is sensitive. In this condition, occurrence of secondary accident is random and sensitive with initial condition. Therefore, reminding drivers existence of accident scene, improving drivers safety consciousness, restricting overloading vehicles and improving traffic environment may avoid secondary traffic accident.

\section{Conclusion}

As a kind of special traffic accident, secondary traffic accident may cause more seriouscasualties and property loss. So, its causation and evolution analysis is importante. Based on Duffing vibrator system, we establish chaos model of secondary traffic accident and analyze chaos characteristics of model. It is indicated that the chaos model may be in periodical vibration state and chaos state by simulation. Example analysis shows that the chaos model can be employed to study evolution of secondary traffic accident. Fruther research should be carried on chaotic attractor and coefficients value in chaos model.

\section{References}

[1] Zhang Wen-hui, Deng Hong-xing, Wang Xian-bin and He Yong-ming: Journal of Transportation Systems Engineering and Information Technology, Vol. 10(2010) No.3, p.105.

[2] Zhang Wenhui, Xu Hongguo: Journal of Transport Information and Safety, Vol. 27(2009) No.1, p.89.

[3] Li Song, Liu Lijun, He Guoguang: Journal of Highway and Transportation Research and Development, Vol. 24(2007) No.12, p.104.

[4] Zhang Sheqi, Zhang Guangjun, Lei Ruide: Journal of Desert Research, Vol. 21(2001) No.1, p.72.

[5] Jiang Jinquan, Li Hong: Chinese Journal of Rock Mechanics and Engineering, Vol. 25(2006) No.5, p.889.

[6] Wang Hongjie, Chi Dezhong: International Conference on Intelligent Computation Technology and Automation, Vol. 2 (2009) No.2, p.189.

[7] Liu Jin, Chang Jianxia, Zhang Wenge: International Conference on Genetic and Evolutionary Computing, Vol. (2009), p.39.

[8] P.Halmes: Journal of Statistic Physics, Vol. 20(1979) , p.181. 